International Journal of Pure and Applied Mathematics

Volume $94 \quad$ No. 5 2014, 701-704

ISSN: 1311-8080 (printed version); ISSN: 1314-3395 (on-line version)

url: http://www.ijpam.eu

doi: http://dx.doi.org/10.12732/ijpam.v94i5.8

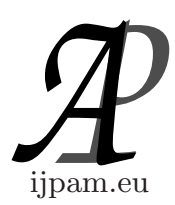

\title{
NEGATIVE BINOMIAL APPROXIMATION FOR GEOMETRIC RANDOM SUMMANDS
}

\author{
K. Teerapabolarn \\ Department of Mathematics \\ Faculty of Science \\ Burapha University \\ Chonburi, 20131, THAILAND
}

\begin{abstract}
This paper determines a bound for the total variation distance between the distribution of random sums of independent geometric random variables and a negative binomial distribution. Two examples have been given to illustrate the result obtained.
\end{abstract}

AMS Subject Classification: 62E17, 60F05, 60G50

Key Words: geometric random variable, negative binomial approximation, random sums, total variation distance

\section{Introduction}

Let $X_{1}, X_{2}, \ldots$ be a sequence of independent geometric random variables, each with $P\left(X_{i}=k\right)=p_{i} q_{i}^{k}, k=0,1, \ldots$, where $q_{i}=1-p_{i}$. Let $N$ be a nonnegative integer-valued random variable and independent of the $X_{i}$ 's. The sum $S_{N}=\sum_{i=1}^{N} X_{i}$ is usually called random sums. Let $N B_{n, p}$ be a negative binomial random variable with parameters $n$ and $p$. For $N=n \in \mathbb{N}$ is fixed, Vellaisamy and Upadhye [2] gave a bound for approximating the distribution of $S_{n}$ by a negative binomial distribution in the form of

Received: March 26, 2014

(c) 2014 Academic Publications, Ltd. url: www.acadpubl.eu 


$$
d_{T V}\left(S_{n}, N B_{n, p}\right) \leq\left(\sum_{i=1}^{n} \frac{q_{i}^{2}}{p_{i}}-\frac{n q^{2}}{p}\right) \min \left\{1, \frac{1}{\sqrt{2 n q e}}\right\},
$$

where $d_{T V}\left(S_{n}, N B_{n, p}\right)=\sup _{A \subseteq \mathbb{N} \cup\{0\}}\left|P\left(S_{n} \in A\right)-P\left(N B_{n, p} \in A\right)\right|$ is the total variation distance between the distribution of $S_{n}$ and the negative binomial distribution with parameters $n$ and $p=1-q=\frac{1}{n} \sum_{i=1}^{n} p_{i}$. Let $\widehat{n}=E(N)$ and $\widehat{q}=1-\widehat{p}=\frac{\lambda}{\widehat{n}}$ where $\lambda=E\left(\lambda_{N}\right)=E\left(\sum_{i=1}^{N} q_{i}\right)$. In this study, we are interested to determine a bound for $d_{T V}\left(S_{N}, N B_{\widehat{n}, \widehat{p}}\right)$, which is in Section 2. In Section 3, two examples have been given to illustrate the desired result. The conclusion of this study is presented in the last section.

\section{Result}

The following theorem presents a bound for the total variation distance between the distributions of $S_{N}$ and $N B_{\widehat{n}, \widehat{p}}$.

Theorem 2.1. For $\lambda_{N}=\sum_{i=1}^{N} q_{i}$ and $\lambda=E\left(\lambda_{N}\right)$, then

$$
\begin{aligned}
d_{T V}\left(S_{N}, N B_{\widehat{n}, \widehat{p}}\right) \leq & \min \left\{1, \frac{0.42888}{\sqrt{\lambda}}\right\} \frac{\lambda^{2}}{\widehat{n}-\lambda}+\min \left\{1, \sqrt{\frac{2}{e \lambda}}\right\} E\left|\lambda_{N}-\lambda\right| \\
& +\min \left\{E\left(\frac{0.42888}{\sqrt{\lambda_{N}}} \sum_{i=1}^{N} \frac{q_{i}^{2}}{p_{i}}\right), E\left(\sum_{i=1}^{N} \frac{q_{i}^{2}}{p_{i}}\right)\right\} .
\end{aligned}
$$

Proof. Let $U_{\lambda}$ be the Poisson random variable with mean $\lambda$. It follows the fact that

$$
d_{T V}\left(S_{N}, N B_{\widehat{n}, \widehat{p}}\right) \leq d_{T V}\left(S_{N}, U_{\lambda}\right)+d_{T V}\left(U_{\lambda}, N B_{\widehat{n}, \widehat{p}}\right)
$$

Teerapabolarn [1] and Vellaisamy and Upadhye [2] showed that

$$
\begin{aligned}
d_{T V}\left(S_{N}, U_{\lambda}\right) \leq & \min \left\{1, \sqrt{\frac{2}{e \lambda}}\right\} E\left|\lambda_{N}-\lambda\right| \\
& +\min \left\{E\left(\frac{0.42888}{\sqrt{\lambda_{N}}} \sum_{i=1}^{N} \frac{q_{i}^{2}}{p_{i}}\right), E\left(\sum_{i=1}^{N} \frac{q_{i}^{2}}{p_{i}}\right)\right\}
\end{aligned}
$$

and

$$
d_{T V}\left(U_{\lambda}, N B_{\widehat{n}, \widehat{p}}\right) \leq \min \left\{1, \frac{0.42888}{\sqrt{\widehat{n} \widehat{q}}}\right\} \frac{\widehat{n} \widehat{q}^{2}}{\widehat{p}}=\min \left\{1, \frac{0.42888}{\sqrt{\lambda}}\right\} \frac{\lambda^{2}}{\widehat{n}-\lambda},
$$


respectively. Hence, the inequality (2.1) is obtained by putting the right hand side of (2.3) and (2.4) to (2.2).

If $X_{i}$ 's are identically distributed, then the following corollary is an immediately consequence of the Theorem 2.1

Corollary 2.1. If $p_{1}=p_{2}=\cdots=p$, then we have the following:

$$
\begin{aligned}
d_{T V}\left(S_{N}, N B_{\widehat{n}, p}\right) \leq & \min \left\{1, \frac{0.42888}{\sqrt{\hat{n} q}}\right\} \frac{\widehat{n} q^{2}}{p}+\min \left\{1, \sqrt{\frac{2}{e \widehat{n} q}}\right\} q E|N-\widehat{n}| \\
& +\min \left\{\frac{0.42888 q^{\frac{3}{2}} E(\sqrt{N})}{p}, \frac{\widehat{n} q^{2}}{p}\right\},
\end{aligned}
$$

and by the fact that $E(\sqrt{N}) \leq \sqrt{\widehat{n}}$, we also obtain

$$
d_{T V}\left(S_{N}, N B_{\widehat{n}, p}\right) \leq \min \left\{1, \frac{0.42888}{\sqrt{\widehat{n} q}}\right\} \frac{2 \widehat{n} q^{2}}{p}+\min \left\{1, \sqrt{\frac{2}{e \widehat{n} q}}\right\} q E|N-\widehat{n}| .
$$

\section{Examples}

This section, we give two examples to illustrate the result in the case of $X_{i}$ 's are identically distributed, which is in the Corollary 2.1 .

Example 3.1. For $n(n \in \mathbb{N})$ is fixed, let $N$ be a positive integer-valued random variable with probability function

$$
P(N=k)= \begin{cases}\frac{1}{2} & , k=n \\ \frac{1}{2} & , k=2 n \\ 0 & , \text { otherwise }\end{cases}
$$

Therefore $\widehat{n}=\frac{3 n}{2}$ and $E|N-\widehat{n}|=\frac{n}{2}$. Let $p_{1}=p_{2}=\cdots=p$, then we have

$$
d_{T V}\left(S_{N}, N B_{\frac{3 n}{2}, p}\right) \leq \min \left\{1, \frac{0.35018}{\sqrt{n q}}\right\} \frac{3 n q^{2}}{p}+\min \left\{1, \sqrt{\frac{0.49051}{n q}}\right\} \frac{n q}{2} .
$$

Example 3.2. Let $N$ be a positive integer-valued random variable with probability function

$$
P(N=n)=\frac{1}{2^{n}}, n=1,2, \ldots,
$$


then we have $\widehat{n}=2$ and $E|N-\widehat{n}|=1$. If $p_{1}=p_{2}=\cdots=p$, then we obtain

$$
d_{T V}\left(S_{N}, N B_{2, \widehat{p}}\right) \leq \min \left\{1, \frac{0.30326}{\sqrt{q}}\right\} \frac{4 q^{2}}{p}+\min \left\{1, \frac{0.36788}{\sqrt{q}}\right\} q .
$$

\section{Conclusion}

In this study, a bound for the total variation distance between the distribution of random sums of independent geometric random variables and an appropriate negative binomial distribution with parameters $\widehat{n}$ and $\widehat{p}$ was obtained. With this bound, it is indicated that the negative binomial with parameters $\widehat{n}$ and $\widehat{p}$ can be used as an approximation of the distribution of random sums of independent geometric random variables when $\widehat{q}=1-\widehat{p}$ is small.

\section{References}

[1] K. Teerapabolarn, Poisson approximation for random sums of geometric random variables, Int. J. Pure Appl. Math., 89 (2013), 35-39.

[2] P. Vellaisamy and N. S. Upadhye, Compound negative binomial approximations for sums of random variables, Probab. Math. Statist., 29 (2009), 205-226. 